\title{
Caracterização química de resíduos da produção de biodiesel compostados com adição mineral
}

\author{
Claudivan C. Lima ${ }^{1}$, Eduardo S. Mendonça ${ }^{2}$, Ivo R. Silva ${ }^{2}$, Luis H. M. Silva ${ }^{3}$ \& Asunción Roig ${ }^{4}$
}

\begin{abstract}
RESUMO
O objetivo do presente trabalho foi avaliar o efeito de diferentes enriquecimentos minerais na compostagem de resíduos provenientes da produção de biodiesel sobre características químicas do produto final. Foram utilizados os seguintes resíduos: bagaço de cana, cinza de bagaço de cana, esterco de galinha poedeira, torta de filtro e farelo de mamona. Estes materiais foram misturados e enriquecidos com fertilizante mineral NPK, pós de rochas de serpentinito + micaxisto e pó de gnaisse, obtendo-se oito compostos orgânicos distintos. Aos 0, 30, 90 e 120 dias de compostagem, essas misturas foram amostrados e realizadas análises de $\mathrm{pH}$, condutividade elétrica, $\mathrm{C}, \mathrm{N}$, cinzas e na última amostragem foram determinados CTC e nutrientes totais. Apenas o composto enriquecido com NPK, sendo o N na forma de sulfato de amônio, atendeu a legislação pertinente a compostos orgânicos, enquanto os compostos contendo torta de filtro e mamona com e sem enriquecimento apresentaram $\mathrm{pH}$ abaixo do limite e os demais apresentaram teores de $\mathrm{N}$ ou $\mathrm{C}$ abaixo do mínimo estabelecido na legislação brasileira. A adição de pós de rochas de serpentinito + micaxisto contribuiu para elevação da CTC dos compostos.
\end{abstract}

Palavra-chave: resíduos orgânicos, compostagem, CTC, legislação brasileira

\section{Chemical characterization of residues from biodiesel production composted with mineral addition}

\begin{abstract}
The aim of this work was to evaluate the effect of different mineral enrichments during composting of biodiesel production residues on chemical characteristics of the end product. The following materials were used: sugar cane bagasse, ashes of sugar cane bagasse, poultry manure, filter cake and castor oil cake. These materials were mixed and enriched with mineral nutrients NPK, serpentinite and micaxist powdered rocks and gneiss powdered rocks, to obtain eight different composts. At 0,30, 90 and 120 days after starting composting, these materials were sampled to measure $\mathrm{pH}$, electrical conductivity, $\mathrm{C}, \mathrm{N}$, ashes, and, at the last sampling, CEC and total nutrients. Only the compost enriched with NPK (with N as ammonium sulphate) comply with the legislation pertinent to compost, while the composts that received filter cake and castor oil cake, with and without, mineral addition presented $\mathrm{pH}$ below the accepted limit and had $\mathrm{C}$ and $\mathrm{N}$ concentrations below the minimum established in the Brazilian legislation. The addition of serpentinite and micaxist powdered rocks contributed to raise the CEC of composts.
\end{abstract}

Key words: organic residue, compost, CEC, Brazilian legislation

\footnotetext{
1 Instituto Federal de Educação Ciência e Tecnologia de Alagoas, Campus Satuba, CEP 57120-000, Satuba, AL. Fone: (82) 326611000. E-mail: claudivanc@yahoo.es

2 Departamento de Solos/UFV, CEP 36570-000, Viçosa, MG. E-mail: esm@ufv.br; ivosilva@ufv.br

3 Departamento de Química/UFV, CEP 36570-000, Viçosa, MG. E-mail: uhen@ufv.br

${ }^{4}$ CEBAS-CSIC, Campus Universitario de Espinardo, Apartado de Correos 164, 30100 Espinardo, Murcia, Spain. E-mail: aroig@cebas.cesic.es
} 


\section{INTRODUÇÃO}

Com a crise energética mundial tem se buscado alternativas renováveis para ampliar a matriz energética que possam substituir, gradativamente, 0 uso de combustíveis fósseis, evitando, com isso, colapsos no abastecimento (Holanda, 2004). No Brasil, o biodiesel (ésteres mono alquila), composto da mistura de óleo vegetal, como o extraido da semente da mamona, e álcool anidro, proveniente da cana-de-açúcar, apresenta-se como excelente alternativa ao óleo diesel, permitindo redução dos níveis de poluição por enxofre em $98 \%$, dos gases de efeito estufa em $78 \%$ e os materiais particulados em 50\% (Holanda, 2004). A L ei no $11.097 / 05$ que introduziu o biodiesel na matriz energética brasileira estabelece os percentuais mínimos de $2 \%$ e $5 \%$ de adição de biodiesel ao óleo diesel mineral, a serem atingidos a partir de janeiro de 2008 e 2013, respectivamente.

Para produção da matéria-prima utilizada na obtenção do biodiesel são gerados grandes volumes de resíduos. No processamento da cana-de-açúcar, por exemplo, são produzidos, para cada tonelada de cana moída, cerca de $30 \mathrm{~kg}$ de torta de filtro, $240 \mathrm{~kg}$ de bagaço de cana e $0,96 \mathrm{~m}^{3}$ de vinhaça (Dematê, 1992). A extração do óleo de mamona, por sua vez, resulta na obtenção de 1,3 toneladas de resíduos conhecidos como torta ou farelo (K iehl, 1995). Estes resíduos, quando dispostos em locais inadequados podem ocasionar poluição do meio, entretanto, quando utilizado na formulação de compostos de uso agrícola, podem contribuir para melhoria da fertilidade do solo e aumento da produtividade das culturas.

A melhoria na qualidade de compostos orgânicos pode ser obtida mediante 0 enriquecimento mineral dos mesmos. Geralmente, as recomendações técnicas para enriquecimento de composto têm enfocado apenas a minimização da perda de $\mathrm{N}$ na forma amoniacal pela adição de fontes de $\mathrm{P}$ ( $\mathrm{K}$ iehl, 1985; Costa, 1985) e sulfato de cálcio (Kiehl, 1985; Prochnow et al., 1995). A adição de fosfatos contribui para formação de compostos estáveis de $\mathrm{N}$ como os fosfatos monoamônico e diamônico e a adição de sulfato de cálcio para formação do sulfato de amônio, possibilitando, deste modo, redução das perdas de $\mathrm{N}$ das medas e a consequente poluição do ar, bem como a obtenção de composto orgânico com maiores teores de $\mathrm{N}$ (K iehl, 1985). A bordagens associando a elevação da CTC de compostos orgânicos à adição mineral, como a realizada por Perez et al. (2005), ainda são escassas na literatura.

Para serem comercializados, os compostos devem apresentar características mínimas constantes na legislação brasileira pertinente. De acordo com a Instrução $\mathrm{N}$ ormativa № 23 de 31 de agosto de 2005, tendo em vista as disposições contidas no Decreto no 4.954 de 14 de janeiro de 2004, que regulamenta a Lei o 6.894 de 16 de dezembro de 1980, os compostos orgânicos deverão ter as seguintes garantias e características: C orgânico total mínimo de $15 \%, \mathrm{~N}$ total mínimo de 1\%, pH mínimo de 6,0, umidade máxima de $50 \%$, relação C:N máxima de 18 , relação $C T C: C$ total de 20 e a soma de NP, NK, PK ou NPK deve ser conforme declarada na embalagem. Já os fertilizantes organo-minerais terão as seguintes garantias e características: C orgânico total com mínimo de 8\%, umidade máxima de 25\%; CTC mínima de $80 \mathrm{mmol}_{\mathrm{c}} \mathrm{kg}^{-1}$, os macronutrientes primários devem apresentar soma de NP, NK, PK ou NPK de $10 \%$, e os macronutrientes secundários soma de $5 \%$, e a soma de micronutrientes de 4\%, cujas tolerâncias destes limites estão explicitadas na referida Instrução Normativa.

0 presente estudo tem por objetivo avaliar o ef eito de diferentes enriquecimentos minerais de composto orgânico sobre características químicas de compostos orgânicos formulados com resíduos da produção de biodiesel.

\section{MATERIAL E MÉTODOS}

\section{Obtenção dos compostos orgânicos}

Foram utilizados diferentes materiais (Tabela 1) para obtenção de oito compostos orgânicos distintos, cujos tratamentos foram constituídos das misturas constantes na Tabela 2. A adição de fertilizantes minerais solúveis nos compostos orgânicos foi realizada de acordo com recomendações para adubação da cana-de-açúcar $\left(80 \mathrm{~kg} \mathrm{ha}^{-1}\right.$ de $\mathrm{P}_{2} \mathrm{O}_{5}, 90 \mathrm{~kg} \mathrm{ha}^{-1}$ de $\mathrm{K}_{2} \mathrm{O}$ e $60 \mathrm{~kg} \mathrm{ha}^{-1}$ de N - CFSEM G, 2002), de modo que com a redução de $2 / 3$ de peso que equivale aproximadamente a $66 \%$ do volume (Peixoto, 1984), resultasse na obtenção de um produto cuja aplicação de $20 \mathrm{M} \mathrm{g} \mathrm{ha}^{-1}$, substituísse a recomendação de NPK para a cultura de cana-de-açúcar. Já a adição dos pós de rochas aos compostos orgânicos foi equivalente à metade do peso seco do esterco de galinha poedeira. As misturas foram acondicionadas em recipientes de $60 \mathrm{~L}$ com perfurações laterais, possibilitando sua aeração. Estes recipientes foram colocados em casa de vegetação pertencente ao D epartamento de Solos da Universidade Federal de Viçosa, Viçosa-M G, no período de agosto de 2003 a dezembro de 2003.

Tabela 1. Composição química dos materiais utilizados na obtenção de diferentes compostos orgânicos

\begin{tabular}{|c|c|c|c|c|c|c|c|}
\hline Caracteristicas $^{1 /}$ & BCA & TF & FM & CBC & EGP & SM & PG \\
\hline $\mathrm{Ca}^{2+}\left(\mathrm{g} \mathrm{kg}^{-1}\right)$ & 10,56 & 11,88 & 11,5 & 26,00 & 11,39 & 75,68 & 15,69 \\
\hline $\mathrm{Mg}^{2+}\left(\mathrm{g} \mathrm{kg}^{-1}\right)$ & 3,19 & 4,68 & 7,0 & 12,20 & 0,65 & 86,87 & 6,67 \\
\hline $\mathrm{K}\left(\mathrm{g} \mathrm{kg}^{-1}\right)$ & 1,42 & 117,11 & 9,6 & 3,52 & nd & 610,34 & 11,71 \\
\hline $\mathrm{Al}^{3+}\left(\mathrm{g} \mathrm{kg}^{-1}\right)$ & 0,39 & 37,19 & - & 9,30 & 0,41 & 21,38 & 7,32 \\
\hline$P\left(g_{k g}^{-1}\right)$ & 0,50 & 9,62 & 17,0 & 12,8 & 1,00 & 0,33 & 9,00 \\
\hline$S\left(g_{k g}^{-1}\right)$ & 0,38 & 0,19 & 2,0 & nd & 0,03 & 1,15 & 5,30 \\
\hline $\mathrm{Fe}\left(\mathrm{mg} \mathrm{kg}^{-1}\right)$ & 1,65 & 171,73 & 12,0 & 877,88 & 202,73 & 38503,03 & 274,37 \\
\hline $\mathrm{Cu}\left(\mathrm{mg} \mathrm{kg}^{-1}\right)$ & $\mathrm{Nd}$ & nd & 75 & 7,15 & 3,45 & 21,47 & 34,98 \\
\hline $\mathrm{Zn}\left(\mathrm{mg} \mathrm{kg}^{-1}\right)$ & $\mathrm{Nd}$ & nd & 120 & nd & 32,54 & 57,89 & 194,02 \\
\hline B (mg kg-1) & $\mathrm{Nd}$ & nd & 90 & nd & nd & 37,13 & 23,26 \\
\hline Mo $\left(\mathrm{mg} \mathrm{kg}^{-1}\right)$ & $\mathrm{Nd}$ & nd & nd & nd & nd & nd & nd \\
\hline $\mathrm{Mn}\left(\mathrm{mg} \mathrm{kg} \mathrm{kg}^{-1}\right)$ & nd & 43,60 & nd & 75,36 & 31,01 & 811,38 & 15,32 \\
\hline $\mathrm{Cr}\left(\mathrm{mg} \mathrm{kg}^{-1}\right)$ & 1,26 & nd & nd & 35,25 & 10,91 & 231,81 & 15,5 \\
\hline $\mathrm{Cd}\left(\mathrm{mg} \mathrm{kg}^{-1}\right)$ & nd & nd & nd & nd & nd & 4,27 & nd \\
\hline $\mathrm{Pb}\left(\mathrm{mg} \mathrm{kg}^{-1}\right)$ & nd & nd & 31 & nd & nd & 0,75 & nd \\
\hline $\mathrm{Ni}\left(\mathrm{mg} \mathrm{kg}^{-1}\right)$ & nd & 0,22 & 382 & 181,83 & 35,25 & $9.014,34$ & 12,77 \\
\hline C Total $\left(\mathrm{g} \mathrm{kg}^{-1}\right)$ & 542,3 & 475,0 & 507,5 & - & 250,8 & - & - \\
\hline N Total $\left(\mathrm{g} \mathrm{kg}^{-1}\right)$ & 14,9 & 21,9 & 46,2 & - & 12,7 & - & - \\
\hline
\end{tabular}

1/Digestão nitroperclórica e determinação de íons metálicos em ICP-OES; nd: não detectado; BCA: bagaço de cana-de-açúcar; TF: torta de filtro; FM: farelo de mamona; CBC: cinza de bagaço de cana; EGP: esterco de galinha poedeira; SM: pó de serpentinito + micaxisto; PG: pó de gnaisse 
Tabela 2. Compostos orgânicos formulados com diferentes materiais e enriquecimentos minerais

\begin{tabular}{clc}
\hline $\begin{array}{c}\text { Composto } \\
\text { orgânico }\end{array}$ & Propoposção \\
CS & $\begin{array}{l}\text { bagaço de cana + cinza de bagaço de cana + } \\
\text { esterco de galinha poedeira }\end{array}$ & $3: 3: 2$ \\
UR & $\mathrm{CS}+\mathrm{NPK}^{*}$ (N: uréia) & $3: 3: 2$ \\
$\mathrm{SA}$ & $\mathrm{CS}+\mathrm{NPK}^{*}$ (N: sulfato de amônio) & $3: 3: 2$ \\
$\mathrm{SM}$ & $\mathrm{CS}+$ micaxisto + serpentinita (em pó) & $3: 3: 2\left(+62,5 \mathrm{~kg} \mathrm{t}^{-1}\right)$ \\
$\mathrm{FN}$ & $\mathrm{CS}+$ fosfato natural & $3: 3: 2\left(+62,5 \mathrm{~kg} \mathrm{t}^{-1}\right)$ \\
TF & bagaço de cana + torta de filtro & $2: 1$ \\
$\mathrm{M}+\mathrm{G}$ & bagaço de cana + farelo de mamona + pó de & $2: 1\left(+62,5 \mathrm{~kg} \mathrm{t}^{-1}\right)$ \\
$\mathrm{M}-\mathrm{G}$ & gnaisse & $2: 1$ \\
\hline
\end{tabular}

* Recomendação para adubação da cana-de-açúcar: $80 \mathrm{~kg} \mathrm{ha}^{-1} \mathrm{P}_{2} \mathrm{O}_{5} ; 90 \mathrm{~kg} \mathrm{ha}^{-1} \mathrm{~K}_{2} \mathrm{O}$ and $60 \mathrm{~kg} \mathrm{ha}^{-1} \mathrm{~N}$

Os tratamentos foram distribuídos em esquema de parcelas subdivididas com 8 compostos na parcela principal e 4 parcelas subdivididas no tempo, formadas pela amostragem do material, sendo dispostos em blocos casualizados, com três repetições. A amostragem dessas misturas foi realizada, seguindo-se o princípio do quarteamento, aos 0, 30, 90 e 120 dias de compostagem, e posteriormente procedidas análises químicas das mesmas.

\section{Caracterização durante o processo de compostagem}

Foram determinados condutividade elétrica em água na relação 1:1 (Simard et al., 1988), pH em $\mathrm{CaCl}_{2}$ 0,01 mol L-1, matéria seca com base na perda de peso a temperatura de $105^{\circ} \mathrm{C}$ (Topp, 1993) e teor de cinzas por ignição em mufla a $550^{\circ} \mathrm{C}$ por $1 \mathrm{~h}, \mathrm{C}$ orgânico total (Yeomans \& B remner, 1988), N total pelo método K jeldahl, de acordo com Miller \& Keeney (1982).

As determinações dos teores de nutrientes dos compostos, após digestão nitroperclórica (M iyazawa et al., 1999), foram realizadas por meio de espectrometria de emissão óptica em plasma induzido (ICP-OES) e a CTC dos compostos, determinada pela saturação do complexo de troca com solução de cloreto de bário ( $L$ ax et al., 1986), foram realizadas apenas na última amostragem.

\section{Análise estatística}

Os dados obtidos foram submetidos à análise de variância, correlação linear simples de Pearson, teste de média para a variável qualitativa "CTC" e análise de regressão, utilizando-se o programa SAEG (Sistema de A nálise Estatísticas e Genéticas), da Universidade Federal de Viçosa (FUNARBE, 1993).

\section{RESULTADOS E DISCUSSÃO}

\section{pH}

O comportamento do $\mathrm{pH}$ ao longo do processo de compostagem definiu dois grupos de compostos distintos de acordo com sua formulação: os contendo $B C+C B C+E G P$ e os contendo BC + TF ou FM (Figura 1). Os tratamentos do primeiro grupo de compostos (CS, UR, SM e FN) apresentaram tendência de elevação do pH, seguindo-se de redução até sua estabilização em torno de pH 7,0, tal como observado em outros trabalhos em que se utilizaram diferentes tipos de resíduo (Bernal et al., 1998; Tejada et al., 2001). Um conjunto de fatores contribui para alterações do pH ao longo do processo de compostagem. Nesse processo, há formação de ácidos solúveis no início da compostagem, os quais são convertidos a dióxido de carbono pela ação microbiana (lyengar \& Bhave, 2005). À medida que este processo se desenvolve o pH do composto se eleva devido a maior concentração de bases que contribui indiretamente para obtenção de um material mais alcalino (Kiehl, 2002).

$\mathrm{N}$ o caso dos tratamentos $S A, M+G, M-G$ e TF que se mantiveram com $\mathrm{pH}$ ácido durante todo o processo, foram afetados por outros fatores. A não alteração do $\mathrm{pH}$ do tratamento SA mantendo-se ácido pode ser atribuída à reação ácida da oxidação do sulfato de amônio, liberando $\mathrm{H}^{+}$para o meio (Alavarez, et al., 1994). A el evação da acidez nos tratamentos $M+G$, $M-G$ e TF ao longo do processo de compostagem pode ser conseqüência do processo de nitificação (M oreira \& Siqueira, 2002). Como os referidos tratamentos apresentam elevados teores de $\mathrm{N}$ orgânico, o mesmo é transformado inicialmente em amônio $\left(\mathrm{NH}_{4}{ }^{+}\right)$pela ação das nitrosomonas, e em seguida em $\mathrm{NO}_{2}$ - pela ação predominante das nitrobactérias, 0 qual se converte rapidamente a nitrato, sendo este o produto final da degradação do N orgânico (Sanchez-M onedero et al., 2001; $\mathrm{K}$ iehl, 2002). O corre que, quando a amônia $\left(\mathrm{NH}_{4}{ }^{+}\right)$é oxidada a $\mathrm{NO}_{3}{ }^{-}$, verifica-se produção líquida de $2 \mathrm{H}^{+}$e, consequentemente, há abaixamento do pH. A ausência de inoculantes como o esterco de galinha poedeira pode também contribuir para esta redução do pH (K iehl, 2002).

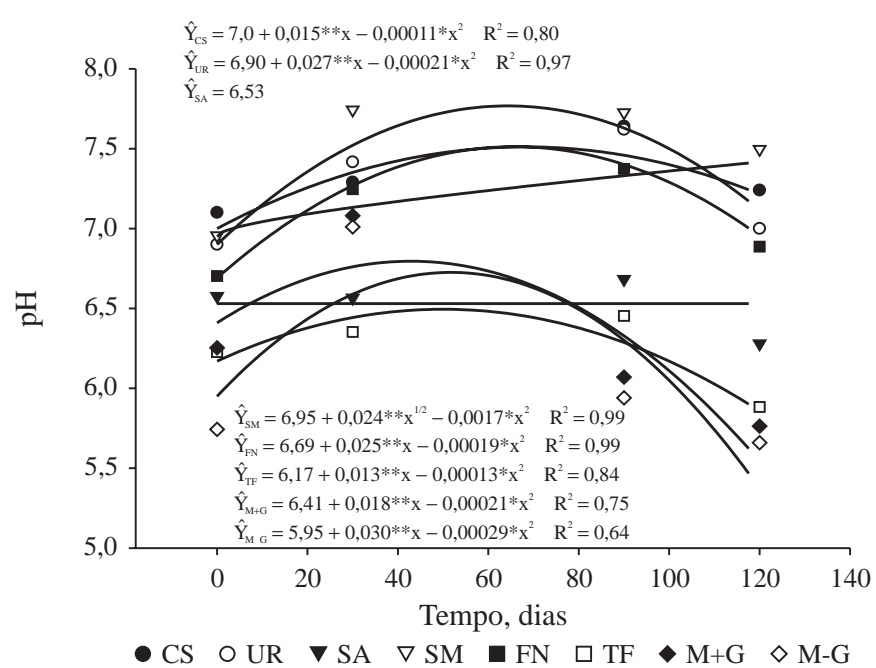

CS: composto simples; UR: CS + NPK, sendo N: uréia; SA: CS + NPK, sendo N: sulfato de amônio; SM: CS + pó de rocha serpentinito + micaxisto; FN: CS + fosfato natural; TF: torta de filtro + bagaço de cana; $M+G$ : farelo de mamona + bagaço de cana + pó de gnaisse; M-G: farelo de mamona + bagaço de cana.

Figura 1. pH de compostos orgânicos formulados com diferentes materiais e enriquecimentos minerais, durante o processo de compostagem

Embora o tratamento UR tenha sido enriquecido com uréia - $\mathrm{CO}\left(\mathrm{NH}_{2}\right)_{2}$, a mesma não foi efetiva na acidificação do meio, pois a uréia ao entrar em contato com a urease dos resíduos orgânicos sofre hidrólise formando $\left(\mathrm{N} \mathrm{H}_{4}\right)_{2} \mathrm{CO}_{3}$ que 
contribui para el evação do $\mathrm{pH}$ ao mesmo tempo que favorece a liberação de amônia $\left(\mathrm{NH}_{3}\right)$, conforme observaram Fernandes et al. (1993) em composto à base de lodo de esgoto enriquecido com uréia.

\section{Condutividade elétrica}

Em todos os tratamentos verificou-se el evação da CE durante o processo de compostagem (Figura 2). A mineral ização dos compostos orgânicos acarreta aumento da concentração de sais solúveis (Campbell et al., 1997). No início da compostagem é possível que o enriquecimento mineral com fertilizantes minerais (SA e UR) tenha contribuído para maior elevação da CE em relação aos demais tratamentos. Entretanto, no decorrer do processo de compostagem, a el evação da CE pode ter sido controlada pela mineral ização da M O, sobretudo pelo processo de nitrificação do $\mathrm{N}$ orgânico que resulta na elevação do teor de nitrato, conforme observaram Sanchez-M onedero et al. (2001) e Rekha et al. (2005). Embora apresentem elevados teores de N (Tabela 3), os tratamentos $M+G$ e $M-G$ e, principalmente, o TF apresentaram elevação menos pronunciada da condutividade elétrica. Isso se deve ao fato de estes compostos não apresentarem em suas respectivas formulações esterco aminal, o qual contribui significativamente com a elevação da CE devido ao alto nível de ionização dos sais presentes nestes materiais, principalmente o cloreto de sódio e 0 cloreto de potássio (Watson, 2006).

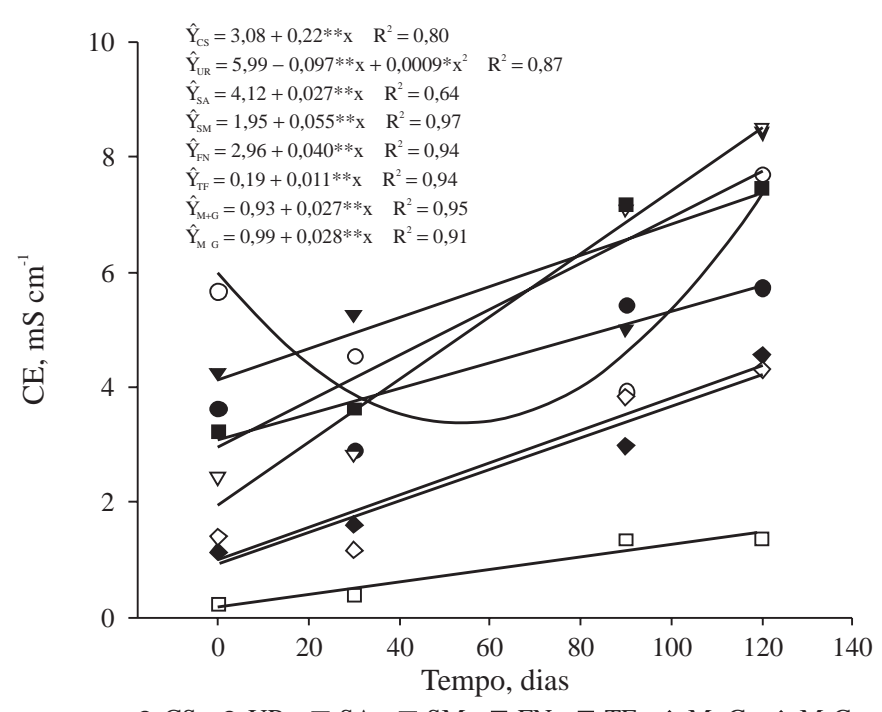

CS: composto simples; UR: CS + NPK, sendo N: uréia; SA: CS + NPK, sendo N: sulfato de amônio; SM: CS + pó de rocha serpentinito + micaxisto; FN: CS + fosfato natural; TF: torta de filtro + bagaço de cana; $M+G$ : farelo de mamona + bagaço de cana + pó de gnaisse; M-G: farelo de mamona + bagaço de cana.

Figura 2. Condutividade elétrica de compostos orgânicos formulados com diferentes materiais e enriquecimentos minerais, durante processo de compostage

\section{Carbono orgânico total e nitrogênio total}

Em geral, o teor de C orgânico durante o processo de compostagem diminuiu e $\mathrm{O}$ de $\mathrm{N}$ total aumentou (Figuras 3 e 4). Isso é conseqüência das perdas de $\mathrm{C}$ na forma de $\mathrm{CO}_{2}$ durante a compostagem e manutenção dos teores de $\mathrm{N}$ neste processo promovendo o seu aumento relativo. A ação dos microrganis- mos sobre a decomposição e humificação da matéria orgânica resulta como produto final dióxido de carbono, água, substâncias húmicas estabilizadas, sais inorgânicos, além do desprendimento de energia térmica (Biddlestone $\&$ Gray, 1985; Hao et al., 2004), concentrando assim o N no composto orgânico.

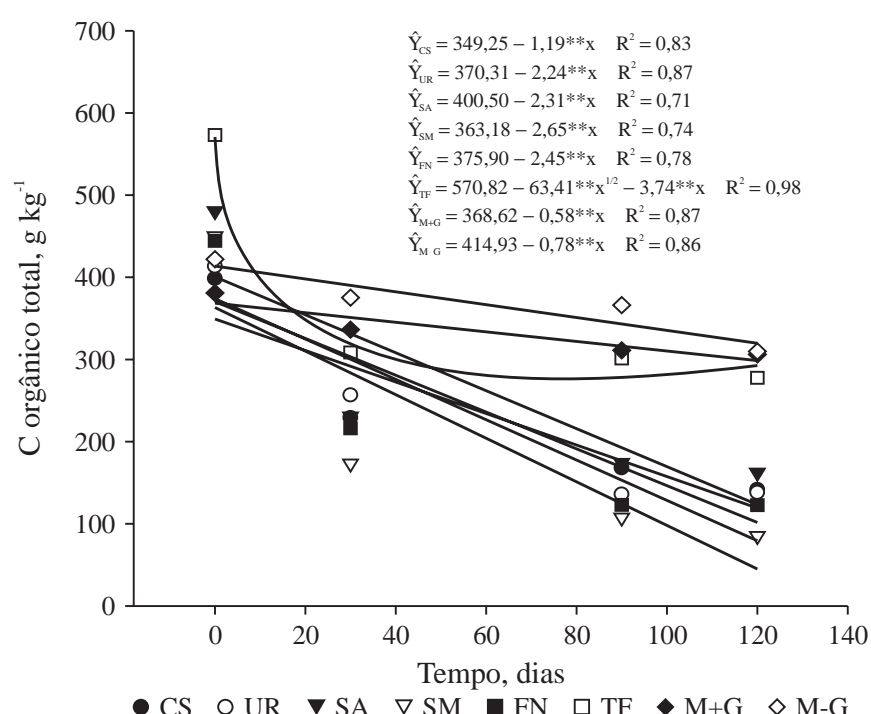

CS: composto simples; UR: CS + NPK, sendo N: uréia; SA: CS + NPK, sendo N: sulfato de amônio SM: CS + pó de rocha serpentinito + micaxisto; FN: CS + fosfato natural; TF: torta de filtro + bagaço de cana; $\mathrm{M}+\mathrm{G}$ : farelo de mamona + bagaço de cana + pó de gnaisse; M-G: farelo de mamona + bagaço de cana.

Figura 3. Teor de carbono orgânico total de compostos orgânicos formulados com diferentes materiais e enriquecimentos minerais, durante o processo de compostagem

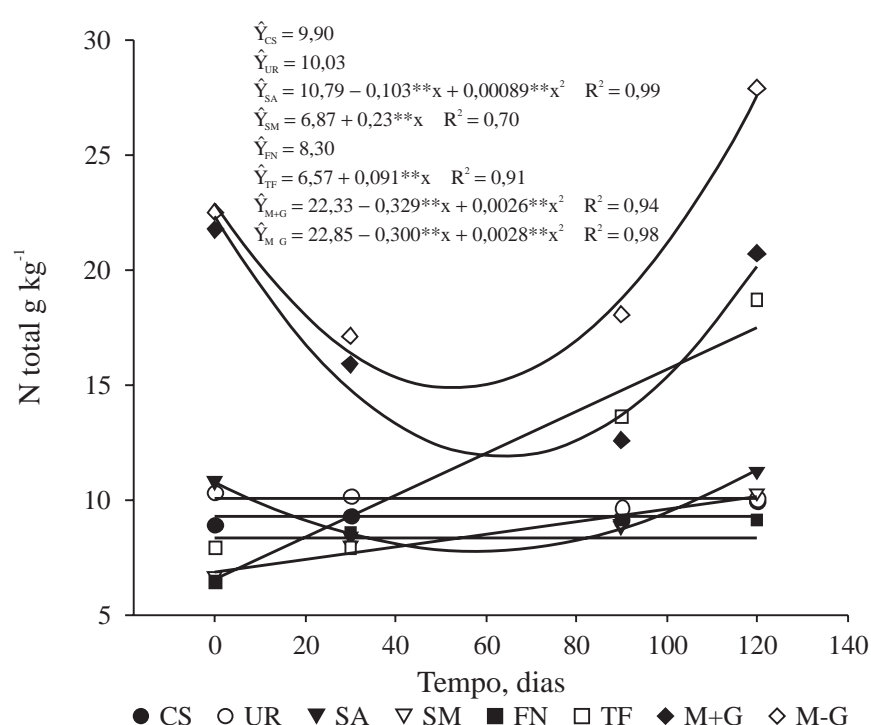

CS: composto simples; UR: CS + NPK, sendo N: uréia; SA: CS + NPK, sendo N: sulfato de amônio; SM: CS + pó de rocha serpentinito + micaxisto; FN: CS + fosfato natural; TF: torta de filtro + bagaço de cana; $\mathrm{M}+\mathrm{G}$ : farelo de mamona + bagaço de cana + pó de gnaisse; $\mathrm{M}-\mathrm{G}$ : farelo de mamona + bagaço de cana.

Figura 4. Teor de nitrogênio total de compostos orgânicos formulados com diferentes materiais e enriquecimentos minerais, durante o processo de compostagem

Índices de avaliação da maturidade dos compostos orgânicos

$O$ índice de maturidade do composto, dado pela relação $C: N$, ficou entre 8,3 e 15,9 no final do processo de compostagem, 
indicando que os compostos encontravam-se praticamente estáveis após 120 dias de compostagem (Figura 5). Segundo H ortenstine \& Rothwell (1973), K ropisz \& Wojciechowski (1978) e Chanyasak \& Kubota (1981), a relação C:N abaixo de 15 expressa estabilidade do composto. Contudo, de acordo com Iglesias-J imenez \& Perez-García (1992), uma relação abaixo de 12 indica alto grau de maturidade do composto. Por outro Iado, Hue \& Liu (1995), Harada \& Inoko (1980) e Harada et al. (1981), afirmam que esta relação pode ser influenciada pela presença de $\mathrm{N}$ amoniacal proveniente de esterco de galinha ou pela adição de uréia a compostos crus.

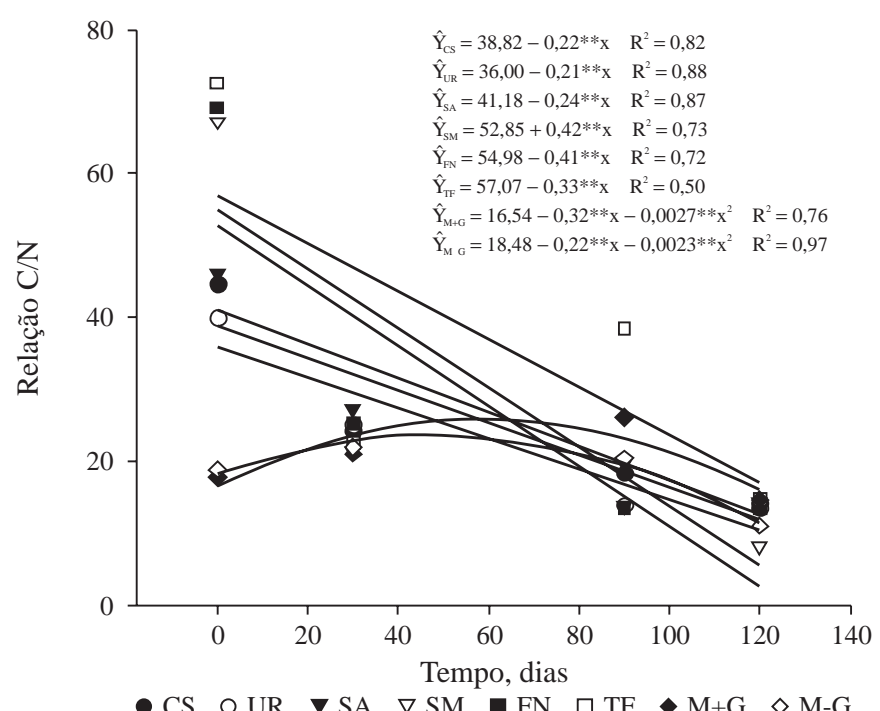

CS: composto simples; UR: CS + NPK, sendo N: uréia; SA: CS + NPK, sendo N: sulfato de amônio SM: CS + pó de rocha serpentinito + micaxisto; FN: CS + fosfato natural; TF: torta de filtro + bagaço de cana; $\mathrm{M}+\mathrm{G}$ : farelo de mamona + bagaço de cana + pó de gnaisse; M-G: farelo de mamona + bagaço de cana.

Figura 5. Relação C:N de compostos orgânicos formulados com diferentes materiais e enriquecimentos minerais, durante o processo de compostagem

\section{Características químicas dos compostos orgânicos}

0 enriquecimento mineral da mescla de materiais para obtenção do composto orgânico não garantiu um produto rico em NPK, no caso dos tratamentos UR e SA (Tabela 3), sobretudo em relação ao N . É bem provável que parte destes fertilizantes tenha se perdido via lixiviação quando do umedecimento dos materiais compostados. Periodicamente, adicionava-se a mesma quantidade de água em todos os compostos quando os mesmos se encontravam aparentemente com baixa umidade. A pós umedecimento dos materiais compostados, observou-se, por vezes, lixiviação de parte da lâmina de água aplicada.

A o se proceder ao primeiro revolvimento, aos 15 dias depois de montado o experimento, verificou-se acúmulo de água na parte inferior do recipiente. Por essa razão, nos umedecimentos seguintes procurou-se evitar lixiviações. À medida que o material orgânico foi se humificando, a massa compostada já apresentava aspecto mais uniforme quanto a sua umidade.

A penas o composto SA, atendeu as normas estabelecidas na legislação brasileira (Instrução N ormativa no 23 de 31 de agosto de 2005) que dispõe sobre especificações e as garantias de fertilizantes orgânicos simples, mistos, compostos, organominerais e biofertilizantes de uso agrícola, comerci-
Tabela 3. Composição química de compostos orgânicos obtidos com diferentes materiais e enriquecimentos minerais

\begin{tabular}{|c|c|c|c|c|c|c|c|c|}
\hline Característica & CS & UR & SA & SM & FN & TF & $M+G$ & M-G \\
\hline $\mathrm{pH}-\mathrm{CaCl}_{2}{ }^{1 /}$ & 7,24 & 7,00 & 6,28 & 7,50 & 6,89 & 5,88 & 5,66 & 5,76 \\
\hline $\begin{array}{l}\text { Cinzas } \\
\left(\text { dag kg }^{-1}\right)\end{array}$ & 71,76 & 74,18 & 74,42 & 76,42 & 76,24 & 41,61 & 31,98 & 16,07 \\
\hline $\begin{array}{l}\mathrm{Ca}^{2+} \\
\left(\mathrm{g} \mathrm{kg}^{-1}\right)^{2 /}\end{array}$ & 71,13 & 74,69 & 69,51 & 70,30 & 111,36 & 22,15 & 80,77 & 13,16 \\
\hline $\begin{array}{l}\mathrm{Mg}^{2+} \\
\left(\mathrm{g} \mathrm{kg}^{-1}\right)^{2 /}\end{array}$ & 5,59 & 5,62 & 5,13 & 21,24 & 5,48 & 1,94 & 11,07 & 5,81 \\
\hline $\mathrm{K}\left(\mathrm{g} \mathrm{kg}^{-1}\right)^{2 /}$ & 110,16 & 107,50 & 96,30 & 204,70 & 322,13 & 478,49 & 59,45 & 76,24 \\
\hline $\mathrm{Al}^{3+}\left(\mathrm{g} \mathrm{kg}^{-1}\right)^{2 /}$ & 9,77 & 8,73 & 7,96 & 13,35 & 9,58 & 49,23 & 5,21 & 6,39 \\
\hline$\left(\mathrm{g} \mathrm{kg}^{-1}\right)^{2 /}$ & 9,93 & 12,21 & 11,50 & 9,05 & 22,84 & 6,54 & 3,58 & 5,96 \\
\hline$\left(\mathrm{g} \mathrm{kg}^{-1}\right)^{2 /}$ & 2,94 & 3,44 & 5,59 & 2,59 & 2,89 & 3,48 & 3,54 & 3,44 \\
\hline $\mathrm{Fe}\left(\mathrm{g} \mathrm{kg}^{-1}\right)^{2 /}$ & 7,20 & 6,70 & 5,82 & 12,67 & 20,21 & 29,69 & 3,77 & 4,41 \\
\hline $\begin{array}{l}\mathrm{Cu} \\
\left.(\mathrm{mg} \mathrm{kg})^{-1}\right)^{2 /}\end{array}$ & 50,08 & 63,32 & 57,46 & 58,44 & 56,01 & 48,16 & 24,21 & 36,68 \\
\hline $\mathrm{Zn}\left(\mathrm{mg} \mathrm{kg}^{-1}\right)^{2 /}$ & 326,29 & 281,90 & 284,64 & 249,66 & 276,17 & 145,52 & 109,16 & 158,95 \\
\hline$\left(\mathrm{mg} \mathrm{kg}^{-1}\right)^{2 /}$ & nd & nd & nd & nd & 38,60 & 4,21 & nd & nd \\
\hline $\begin{array}{l}\text { Mo } \\
\left(\mathrm{mg} \mathrm{kg}^{-1}\right)^{2 /}\end{array}$ & 1,38 & 0,13 & 0,25 & nd & nd & nd & nd & nd \\
\hline $\begin{array}{l}\mathrm{Mn} \\
\left(\mathrm{mg} \mathrm{kg}^{-1}\right)^{2 /}\end{array}$ & 472,95 & 418,51 & 394,51 & 503,63 & 596,61 & 892,62 & 316,07 & 330,24 \\
\hline $\mathrm{Cr}\left(\mathrm{mg} \mathrm{kg}^{-1}\right)^{2 /}$ & 47,19 & 29,27 & 46,44 & 93,40 & 63,10 & 33,65 & 8,30 & 11,88 \\
\hline $\begin{array}{l}\mathrm{Cd} \\
\left(\mathrm{mg} \mathrm{kg}^{-1}\right)^{2 /}\end{array}$ & 0,20 & 0,16 & 0,16 & 0,99 & 2,07 & 3,15 & nd & 0,01 \\
\hline $\mathrm{Pb}\left(\mathrm{mg} \mathrm{kg}^{-1}\right)^{2 /}$ & 1,44 & 1,60 & 0,55 & 0,42 & 6,74 & 4,48 & 0,40 & 1,77 \\
\hline $\mathrm{Ni}\left(\mathrm{mg} \mathrm{kg}^{-1}\right)^{2 /}$ & 69,30 & 93,65 & 89,93 & 1663,95 & 5100,05 & 209,20 & 16,17 & 39,71 \\
\hline $\begin{array}{l}\text { C.E. } \\
\left(\mathrm{mS} \mathrm{cm}^{-1}\right)^{3 /}\end{array}$ & 5,72 & 7,70 & 8,41 & 8,50 & 7,44 & 1,26 & 4,29 & 4,55 \\
\hline $\begin{array}{l}\text { C Total } \\
\left(\mathrm{g} \mathrm{kg}^{-1}\right)\end{array}$ & 141,51 & 137,94 & 162,34 & 85,28 & 122,81 & 277,72 & 306,11 & 309,77 \\
\hline $\begin{array}{l}\text { N Total } \\
\left(\mathrm{g} \mathrm{kg}^{-1}\right)\end{array}$ & 8,9 & 10,0 & 11,2 & 10,3 & 9,1 & 18,7 & 20,7 & 27,9 \\
\hline Relação C:N & 15,9 & 13,8 & 14,5 & 8,3 & 13,5 & 14,8 & 14,8 & 11,1 \\
\hline $\begin{array}{l}\text { CTC } \\
\left(\mathrm{cmol}_{\mathrm{c}} \mathrm{dm}^{-3}\right)^{4 /}\end{array}$ & $41,66^{B}$ & $42,77^{\mathrm{B}}$ & $37,78^{\mathrm{B}}$ & $61,82^{\mathrm{A}}$ & $35,77^{\mathrm{B}}$ & $61,39^{A}$ & $43,12^{\mathrm{B}}$ & В $43,66^{\mathrm{B}}$ \\
\hline CTC:Ct & 2,94 & 3,10 & 2,33 & 7,25 & 2,91 & 2,21 & 1,41 & 1,41 \\
\hline
\end{tabular}

${ }^{1}$ Relação sólido-solução 1:5; ${ }^{2 /}$ Digestão nitroperclórica, leitura em ICP-OES; ${ }^{3 / C o n d u t i v i d a d e ~ E l e ́ t r i c a ~}$ (Simard et al., 1988) op cite; ${ }^{4 / C a p a c i d a d e ~ d e ~ T r o c a ~ C a t i o ̂ n i c a ~(L a x ~ e t ~ a l ., ~ 1986) ~ o p ~ c i t e ; ~ n d: ~ n a ̃ o ~}$ detectado; Letras acima dos valores de CTC referem-se à comparação de médias entre os tratamentos; médias seguidas de mesma letra não diferem significativamente em nível de $5 \%$ de probabilidade pelo teste Tukey; CS: composto simples; UR: CS enriquecido com NPK, cujo N: uréia; SA: CS enriquecido com NPK, cujo N: sulfato de amônio; SM: CS enriquecido com pó de rocha serpentinito + micaxisto; FN: CS enriquecido com fosfato natural; TF: torta de filtro + bagaço de cana; M+G: farelo de mamona + bagaço de cana + pó de gnaisse; M-G: farelo de mamona + bagaço de cana.

alizados no Brasil. Já os compostos $T F, M+G$ e $M-G$ apresentaram pH abaixo do limite legal e os demais apresentaram teores de $\mathrm{N}$ ou $\mathrm{C}$ abaixo do mínimo estabelecido na referida legislação. 0 elevado conteúdo de cinza destes contribuiu para redução relativa da concentração de tais elementos, denotando que a adição de cinzas de bagaço de cana na dose aplicada não é uma boa recomendação. A relação CTC:Ct de 20, estabelecida na legislação brasileira, cuja CTC está expressa em $\mathrm{mmol}_{\mathrm{c}} \mathrm{dm}^{-3}$, apresenta-se ligeiramente superior a consagrada como referência do processo de humificação de compostos que é de 1,7 (Roig et al., 1988) a qual tem CTC expressa em $\mathrm{cmol}_{\mathrm{C}} \mathrm{dm}^{-3}$, o que equivale, na legislação brasileira, a uma relação CTC:Ct de 17.

Os compostos orgânicos enriquecidos atenderam todas as condições legais para serem considerados fertilizantes organo-minerais, inclusive a CTC que ficou acima de $80 \mathrm{mmol}_{\mathrm{c}} \mathrm{kg}^{-1}$ ou $8 \mathrm{cmol}_{\mathrm{c}} \mathrm{kg}^{-1}$. Contudo, I glesias-J imenez $\&$ 
Perez-García (1992) e Solano et al. (2001), sugeriram como indicação de maturidade de composto orgânico CTC acima de $67 \mathrm{cmol}_{\mathrm{c}} \mathrm{kg}^{-1}$. Provavel mente a adição de cinza na formulação dos mesmos contribuiu para que os valores da CTC ficassem abaixo deste limite, uma vez que a CTC dos compostos CS, UR, SA, SM, FN, TF, isentos de cinzas, ficou acima do referido limite:147,52, 165,65, 147,69, 262,17, $150,55,105,14 \mathrm{cmol}_{\mathrm{c}} \mathrm{kg}^{-1}$ de $\mathrm{M} \mathrm{O}$, respectivamente, a CTC dos compostos $M+G$ e $M-G$, com 63,39 e 50,02 $\mathrm{cmol}_{\mathrm{c}} \mathrm{kg}^{-1}$ de $M 0$, respectivamente, ficou abaixo.

A s características químicas dos compostos dependem bastante do material utilizado na compostagem e do enriquecimento mineral aplicado. Nesse particular, houve grande contribuição dos pós de rochas para elevação dos teores de $\mathrm{P}$, $\mathrm{K}$, Ca e Mg, (Tabela 1). Contudo, a disponibilidade destes para a planta depende, em parte, da dinâmica de decomposição dos materiais orgânicos e da própria solubilidade dos pós de rocha. Desta forma, não é possível garantir que o enriquecimento mineral dos compostos UR e SA, por exemplo, enriquecidos com base na recomendação da cultura da cana-de-açúcar, irão atender a demanda de nutrientes da cultura, ou que os tratamentos SM e FN enriquecidos com pós de rocha suprirão as demandas das plantas cultivadas. Para tanto, se faz necessário avaliar agronomicamente 0 potencial de utilização destes compostos orgânicos enriquecidos comparados à adubação química convencional.

\section{CONCLUSÕES}

1. Verificou-se que apenas o composto SA, atendeu a legislação brasileira (Instrução N ormativa no 23 de 31 de agosto de 2005) pertinente a compostos orgânicos, enquanto os $T F, M+G$ e $M-G$ apresentaram $\mathrm{pH}$ abaixo do limite legal e os compostos CS, UR, SM e FN apresentaram teores de N ou $C$ abaixo do mínimo estabelecido na legislação.

2. Os compostos orgânicos enriquecidos atenderam todas as condições legais para serem considerados fertilizantes organo-minerais, de acordo com a lesgislação brasileira.

3. Os el evados teores de cinza dos compostos CS, UR, SA, $\mathrm{SM}$ e $\mathrm{FN}$ contribuíram para redução relativa da concentração de $\mathrm{C}, \mathrm{N}$, bem com dos teores de CTC destes compostos.

4. A adição de pós de rochas de serpentinito + micaxisto aos compostos orgânicos à base de bagaço de cana, cinza de bagaço de cana e esterco de galinha poedeira contribuiu para elevação da CTC dos mesmos.

5. Os pós de rochas contribuiram para elevação dos teores de $\mathrm{P}, \mathrm{K}, \mathrm{Ca}$ e $\mathrm{M} \mathrm{g}$, contudo, a disponibilidade destes para a planta depende da dinâmica de decomposição dos materiais orgânicos e da solubilidade dos mesmos.

\section{AGRADECIMENTOS}

Os autores agradecem ao CNPq (Conselho Nacional de Desenvolvimento Científico e Tecnológico) e a FAPEAL (Fundação de A mparo à Pesquisa do Estado de A lagoas) pelo suporte financeiro para realização desta pesquisa.

\section{LITERATURA CITADA}

Alvarez, V. V. H.; M ello, J. W. V. de; Dias, L. E. Curso de fertilidade e manejo de solos. Brasília: A BEAS. 61 p. 1994. Módulo 04: A cidez do Solo.

B ernal, M. P.; Paredes, C.; Sanchez-M onedero, M. A.; Cegarra, J. $M$ aturity and stability parameters of composts prepared with a wide range of organic wastes. Bioresource Technology, v.63, n.1, p. 91-99, 1998.

Biddlestone, A. J .; Gray, K. R. Composting. In: M 00-Y OUNG, M. (ed.). Comprehensive Biotecnology, v. 4. Pergamon Press, Oxford, 1985, $1059 \mathrm{p}$.

Campbell, A. G.; Folk, R. L.; Tripepi, R. Wood ash as amendment in municipal sludge and yard waste composting processes. Compost Science $\&$ Utilization, v. 5, n. 1, p. 62-63, 1997.

Chanyasak, V.; Kubota, H. Carbon/organic nitrogen ratio in water extracts as measure of compost degradation. Journal of Fermentation Technology, v. 59, n. 3, p. 215-219, 1981.

Comissão de Fertilidade do Solo do Estado de Minas Gerais. Recomendações para uso de corretivos e fertilizantes em $M$ inas Gerais; 50 aproximação. Lavras, 2002, 359 p.

Costa, M. B. B. A dubação orgânica: nova síntese e novo caminho para a agricultura. São Paulo: Ícone, 1985. 100 p.

Dematê, J. L. I. U so A gronômico de Resíduos x Fertilizantes na Cultura da Cana-de-açúcar. In: Reunião Brasileira de Fertilidade do Solo e Nutrição de Plantas, 20, 1992. Piracicaba. Resumos... Piracicaba: Fundação Cargill, 1992, p. 213-252.

Drozd, J.; Jamroz, E.; Licznar, M .; Licznar, S. E.; Weber, J. Organic matter transformation and humic indices of compost maturity satage during composting of municipal solid wastes. Grunwaldzka, v. 53, p. 855-861, 1997.

Fernandes, F.; Pierro, A. C.; Yamamoto, R. Y. Produção de fertilizante orgânico por compostagem do lodo gerado por estações de tratamento de esgotos. Pesquisa A gropecuária Brasileira, v. 28, n. 5, p. 567-574, 1993.

Foy, C. D. Soil chemical factors limiting plant root growth. In: Steward, B. A. (Ed.), A dvance Soil Science, v. 19, Springer Verlag, New York, p. 97-149, 1992.

FUNARBE. SAEG - Sistema para análises estatísticas - versão 5.0. Viçosa: Fundação Arthur Bernardes. 1993. 80p.

Hao, X.; Chang, C.; Larney, F. J. Carbon, nitrogen balances and greenhouse gas emission during cattle feedlot manure composting. Journal of Environmental Quality, v. 33, n. 1, p. 37-44, 2004.

Harada, Y.; Inoko, A. Relationship between cation exchange capacity and degree of maturity of refuse compost. Soil Science and Plant Nutrition, v. 26, p.353-362, 1980.

Harada, Y.; Inoko, A .; Tadaki, M.; Izawa, T. M aturity process of city refuse composting during piling. Soil Science and Plant Nutrition, v. 27, p. 357-364, 1981.

Holanda, A. Biodiesel e Inclusão Social. B rasília, DF : Câmara dos Deputados - Coordenação de Publicações, 2004, 200 p. (Caderno Altos Estudos).

Hortenstine, C. C.; Rothwell, D. F. Pelletized municipal refuse compost as a soil amendment and nutrients sourse for sorghum. Journal of Environmental Quality, v. 2, p. 343-345, 1973.

Hsu, J. H.; L O, S. L. Chemical and spectroscopic analysis of organic matter transformations during of pig manure. Environmental Pollution, v. 104, n. 2, p. 189-196, 1999. 
Hue, N. V.; Liu, J. Predicting compost stability. Compost Science $\&$ Utilization, v. 3, n. 1, p. 8-15, 1995.

I glesias-J imenez, E.; Perez-Garcia, V. Determination or maturity indices for city refuse composts. A griculture, Ecosystems and Environmental, v. 38, n. 4, p. 331-343, 1992.

Iyengar, S. R.; B have, P. P. In-vessel composting of household wastes. Waste M anagement, v. 26, n. 10, p. 1070-1080, 2006.

Kiel, J. K. Fertilizantes Orgânicos. Piracicaba: Ceres, 1985. 492 p.

Kiel, J. K. M anual de Compostagem: M aturação e Qualidade do Composto. Piracicaba: 3a Edição do A utor, 2002. 171 p.

K ropisz, A.; Wojciechowski, J. M utual effects of mineral fertilizers and compost made from municipal wastes on yields and chemical composition of cabbage. Roczniki Nauk Rolniczych, v. 103, n. 2, p. 164-180, 1978.

Lax, A.; Roig, A.; Costa, F. A method for determining the cationexchange capacity of organic materials. Plant and Soil, v. 94, n. 3, p. 349-355, 1986.

M iller, R. H.; K eeney, D. R. M ethods of soil analysis. Part 2: Chemical and microbiological properties. 2.ed. Madison: A merican Society of A gronomy, 1982. 1159p.

M iyazawa, M.; Pavan, M. A .; M uraoka, T.; Carmo, C. A. F. S.; M ello, W. J. A nálise química de tecido vegetal. p. 171-223. In: SILVA, F. C. M anual de A nálises Químicas de Solos, Plantas e Fertilizantes. B rasília: EM BRA PA, 1999, 370 p.

M oreira, F. M. S.; Siqueira, J. O. M icrobiologia e Bioquímica do Solo. Lavras: Editora UFLA, 2002. 626 p. il.

Peixoto, R. T. dos G. Solubilização de fosfato natural durante a compostagem de lixo urbano e sua utilização por feijão e sorgo forrageiro. Rio de J aneiro: UFRRJ, 1984, 235p. Dissertação M estrado.

Perez, B. H.; Lima, C. C.; M endonça, E. S.; Silva, I. R.; Roig, A.; Santos, E. R. D. Influência da natureza dos materiais utilizados e do enriquecimento mineral sobre a CTC de compostos orgânicos. In: Congresso Brasileiro de Ciência do Solo, 30, 2005. Recife: Resumos... Recife: SBCS, UFRPE, 2005, CD Rom.
Prochnow, L. I.; Kiehl, J. C.; Pismel, F. S.; Corrente, J. E. Controlling ammonia losses during manure composting with the addition of phosphogypsum and simple superphosphate. Scientia A gricola, v. 52, n. 2, p.346-349, 1995.

Rekha, P.; Raj, D. S. S.; A parna, C.; B indu, V. H.; A njaneyulu, Y. Bioremediation of contaminated lake sediments and evaluation of maturity índices as indicators of compost stability. J ournal of Environmental Research Public Health, v. 2, n. 2, p. 251262, 2005.

Roig, A.; Lax, A.; Cegarra, J.; Costa, F.; Jernadez, M. T. Cations exchanger capacity as a parameter for measuring the humification degree of manure. Soil Science, v. 146, n. 5, p. 311-316, 1988.

Sanchez-M onedero, M. A.; Roig, A.; Paredes, C.; B ernal, P. Nitrogen transformation by Rutgers system and its effects on $\mathrm{pH}$, EC and maturity of the composting mixtures. Bioresource Technology, v. 78, n. 3, p. 301-308, 2001.

Simard, R. R.; Evans, L. J.; Bares, T. E. Effects of additions of $\mathrm{CaCO}_{3}$ and $\mathrm{P}$ on the soil solution composition of a Podzolic soil. Canadian Journal of Soil Science, Boca Raton: Lewis Publish, v. 68, p. 41-52. 1988.

Solano, M. L.; I riarte, F.; Ciria, P.; N egro, M. J. Performance characteristics of thee aeration systems in the composting of sheep manure and straw. Journal of A gricultural Engineering Research, v. 79, n. 3, p. 317-329, 2001.

Tejada, M.; Dobao, M. M.; Benitez, C.; Gonzalez, J. L. Study of composting of cotton residues. Bioresource Technology, v.79, n.1, p.199-202, 2001.

Topp, G. C. Soil water content. In: Carter, M. R. (Ed.) Soil sampling and methods of analysis. Canadian Society of Soil Science. Boca Raton: Lewis Publish, 1993. p. 541-557.

Watson, M. E. Testing compost. Extenson Tastsheet. http:// ohioline.osu.edu/anr-fact/0015.html. A cessado em 17 de jutho de 2006.

Yeomans, J. C.; B remner, J. M . A rapid and precise method for routine determination of organic carbon in soil. Communications in Soil Science and Plant A nalysis, v. 19, p. 1467-1476, 1988. 